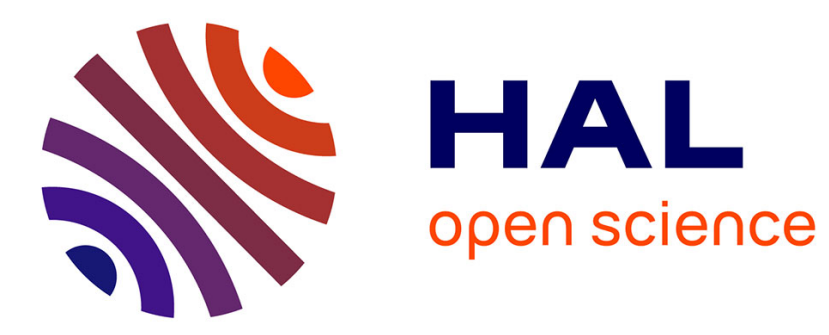

\title{
L'invention du vote secret en Angleterre. Idéologie, intérêt et circulation des arguments
}

Christophe Jaffrelot

\section{To cite this version:}

Christophe Jaffrelot. L'invention du vote secret en Angleterre. Idéologie, intérêt et circulation des arguments. Politix, 1993, 6 (22), pp.43-68. 10.3406/polix.1993.2043 . hal-01009727

HAL Id: hal-01009727

https://hal-sciencespo.archives-ouvertes.fr/hal-01009727

Submitted on 18 Jun 2014

HAL is a multi-disciplinary open access archive for the deposit and dissemination of scientific research documents, whether they are published or not. The documents may come from teaching and research institutions in France or abroad, or from public or private research centers.
L'archive ouverte pluridisciplinaire HAL, est destinée au dépôt et à la diffusion de documents scientifiques de niveau recherche, publiés ou non, émanant des établissements d'enseignement et de recherche français ou étrangers, des laboratoires publics ou privés. 


\title{
L'invention du vote secret en Angleterre. Idéologie, intérêt et circulation des arguments
}

In: Politix. Vol. 6, №22. Deuxième trimestre 1993. pp. 43-68.

\begin{abstract}
The invention of secret vote in England.

Christophe Jaffrelot. [43-68]

By contrast with the early and steady extension of the franchise in Great-Britain, the adoption of the ballot arose many debates. Mostly, they reflected the reluctance front notables towards any restriction of their influence but also the specificity of the utilitarian ideology, on which the radical school of thought (the main agency of the democratisation process) was based : one of the corner stones of Bentham's philosophy being the principale of publicity, on one hand it hampered for some time the demand, by the radicals, of the ballot wich the middle class, their constituency, wanted strongly and on the other hand it provided the opponents to the ballot, conservative whigs as well as tories, with new arguments.
\end{abstract}

\section{Résumé}

L'invention du vote secret en Angleterre.

Christophe Jaffrelot. [43-68].

Par contraste avec le caractère somme toute précoce et régulier de l'extension de la franchise en Grande-Bretagne, l'introduction du vote secret (ballot) fit l'objet de longs débats. Ceux-ci reflétaient en grande partie la réticence des notables envers toute réduction de leur influence mais aussi la spécificité de l'idéologie utilitariste à l'origine du courant Radical, le principal aiguillon de la démocratisation anglaise : l'école de Bentham valorisait en effet au plus haut point le principe de publicité, ce qui d'une part, retarda un peu la mobilisation des radicaux sur le thème du vote secret que revendiquaient les classes moyennes dont ils étaient l'émanation et d'autre part fournit des arguments aux opposants du ballot, whigs conservateurs et tories.

Citer ce document / Cite this document :

Jaffrelot Christophe. L'invention du vote secret en Angleterre. Idéologie, intérêt et circulation des arguments. In: Politix. Vol. 6, N²2. Deuxième trimestre 1993. pp. 43-68.

doi : 10.3406/polix.1993.2043

http://www.persee.fr/web/revues/home/prescript/article/polix_0295-2319_1993_num_6_22_2043 


\section{L'invention du vote secret en Angleterre}

Idéologie, intérêt et circulation des arguments

Christophe Jaffrelot

Centre d'études et de recherches internationales

$\mathrm{L}^{\prime}$

- ANGLETERRE, qui avait amorcé le processus de démocratisation de façon somme toute précoce, n'adopta le vote secret (le ballot) qu'en 1872, alors qu'en France, par exemple, cette procédure était en principe entrée en vigueur depuis la loi de $1849^{1}$. La loi instituant l'isoloir - avant lequel le secret du vote était un fait aléatoire - ne fut cependant approuvée qu'en 1913 après maints débats. Ce caractère tardif s'explique, en grande partie, d'après l'étude d'Alain Garrigou, par l'opposition de notables soucieux d'exercer leur influence sur les électeurs, voire de mener quelque représaille contre les déviants, en tout cas de faire du scrutin une procédure de ratification de l'autorité qu'ils représentent plutôt qu'un choix libre assorti d'un mandat ${ }^{2}$. Tardive, ala loi de 1913 sanctionne en fait une évolution lente et profonde du mode de domination politique qui amène un nouveau type d'entrepreneurs politiques à faire prévaloir ses conceptions et ses intérêts propres au détriment de concurrents dont la résistance visait à maintenir une définition légitime conforme à leurs intérêts et qui avaient dominé aussi longtemps qu'ils dominaient dans les assemblées parlementaires et donc qu'ils avaient pu l'emporter électoralement ${ }^{3}$.

Cette interprétation peut aussi s'appliquer au cas britannique, mais ces conflits d'intérêts ne semblent pas constituer le seul point de départ pertinent. L'idéologie utilitariste élaborée par $\mathrm{J}$. Bentham au début du siècle et d'où procède le principal mouvement démocratique, le courant radical, fournit en effet les premières clés du débat sur le ballot. Le radicalisme deviendra très vite l'idéologie de prédilection des principaux représentants de la classe moyenne, décidés à supplanter l'élite conservatrice, notamment au moyen du vote secret. Et cette dernière s'opposera à celui-ci au nom de ses intérêts. Mais les adversaires du ballot emprunteront leurs arguments les plus décisifs à des principes utilitaristes, en vertu d'une cohérence idéologique et pas seulement de leur intérêt social, comme dans le cas du libéral Gladstone. Les débats autour du ballot dans l'Angleterre du XIXe siècle permettent donc sans doute d'illustrer l'imbrication des facteurs idéologiques et de l'intérêt social dans la revendication du vote secret, la prise en compte de la cohérence idéologique

1. Ce texte procède, lointainement, de deux mémoires réalisés en 1985 et 1986 sous la direction respectivement de Bernard Manin et Jean Leca.

2. Garrigou (A.), -Le secret de l'isoloir., Actes de la recherche en sciences sociales, $n^{\circ} 71-72,1988$,

p. 39.

3. Ibid., p. 23. 
contribuant à expliquer la circulation des arguments du camp des partisans de cette mesure à celui des opposants, et vice versa.

\section{Les ressorts idéologiques et sociaux du mouvement des radicaux pour le ballot}

A soixante-deux ans, Bentham remet en cause son allégeance au torysme lorsqu'il aborde enfin le problème politique dans son Catéchisme de la réforme parlementaire (1810). Mais celle-ci n'incorpore pas la revendication bientôt centrale de l'extension du droit de vote et "la réforme ne présente pas d'avantages directs par son côté démocratique ${ }^{1}$. Immergé dans le milieu "radical» de Westminster, Bentham va cependant évoluer jusqu'à rédiger en 1818 les vingt-six. Résolutions sur la réforme parlementaire que Burdett agrée et propose aux Communes. Surtout, en 1824, il achève le Code constitutionnel dont il a entamé la rédaction en 1820 et exprime sous sa forme la plus articulée l'idéal politique des Utilitaristes. En 1820, cependant, James Mill, réputé pour exprimer clairement la pensée complexe de Bentham, fait paraître son Essay on Government. L'étude parallèle de ces écrits très rapprochés dans le temps permet une appréhension globale de la doctrine politique des Utilitaristes.

\section{La conversion utilitariste à la démocratie et au vote secret}

L'objectif bien connu de l'utilitarisme de Bentham est ale plus grand bonheur du plus grand nombre. Fondamentalement individualiste, Bentham considère que al'intérêt de la communauté est [...] la somme des intérêts des différents membres qui la composent ${ }^{2}$. Ces prémisses incorporent une tension évidente entre deux pôles, l'individu et la société. La conciliation des intérêts de l'un et de l'autre ne peut a priori se vérifier que si celui de l'individu est compatible avec celui de ses concitoyens. Or, cette hypothèse est rejetée par Bentham qui tient pour acquis que les actions humaines sont exclusivement commandées par la poursuite de l'intérêt égoïste. Le procédé par lequel Bentham va s'attacher à résoudre les contradictions entre intérêt privé et intérêt général s'inscrit dans son souci d'édifier une science de la morale et de la législation conforme aux exigences de la Raison.

Le principe de publicité, pilier de l'identification artificielle des intérêts

Il s'agit, une fois acquise la connaissance scientifique de la psychologie humaine, d'agir sur ce qu'on admet être sa tendance la plus sensible - la maximisation du bonheur - pour l'amener a identifier son intérêt propre avec celui de la communauté. Ce processus ne peut s'opérer qu'à partir de lois récompensant ou réprimant l'individu : "Tout l'art du législateur consiste à forcer les hommes par le sentiment de l'amour d'eux-mêmes à être toujours justes envers les autres. Toute l'étude du moraliste consiste à déterminer l'usage qu'on doit faire des récompenses et des peines, et le secours qu'on

1. Halévy (E.), La formation du radicalisme pbilosopbique, vol. 2, L'évolution de la doctrine utilitaire de 1789 à 1815, Paris, Félix Alcan, 1901, p. 203.

2. Parekh (B.), Bentham's political thought, London, Croom Helm, 1973, p. 68. 
peut en tirer pour lier l'intérêt personnel et l'intérêt général"1. Ce dispositif doit donc permettre de promouvoir sans risque d'erreur le plus grand bonheur du plus grand nombre. En matière de droit constitutionnel, le principe de l'identification àrtificielle des intérêts udevient le problème de l'identification artificielle des intérêts des gouvernants et des gouvernés "2 . Les moyens requis pour accomplir ce processus sont recensés avec rigueur par J. Mill qui recherche, en 1820, la forme de gouvernement la plus adéquate.

La solution de la démocratie directe qui impliquerait la constante mobilisation des producteurs ne lui paraît pas permettre un travail cohérent et la critique d'incompétence invalide tout autant un régime aristocratique qui reviendrait surtout à concentrer tous les pouvoirs entre les mains d'une caste indifférente au plus grand nombre. L'argument hypothèque plus encore le recours à la monarchie car "quand un homme est appelé roi, cela ne change pas sa nature lpar essence égoïste]n 3 . Dès lors, l'unique solution réside dans la démocratie représentative. Bentham porte la défiance à un degré extrême puisqu'il se prononce pour des élections d'une fréquence au moins annuelle, "pour assurer plus effectivement l'unité de volonté et d'opinion entre le peuple et ses représentants ${ }^{4}$. Répondant à des considérations très pragmatiques, la conversion à la démocratie opérée par les Benthamites est finalement d'autant plus remarquable que la formule choisie, si elle ne correspond pas à la démocratie directe, vise néanmoins à subordonner le plus possible les représentants à leurs mandants. L'utilitarisme de Bentham constitue donc l'acte de naissance d'un courant démocratique proprement anglais qu'on appellera "radicalisme" et qui contraste avec le courant dit "jacobin", tout en convergeant avec lui. L'essor de la revendication démocratique en Angleterre est indissociable du processus de décolonisation engagé par les Britanniques d'Amérique qui refusent de payer l'impôt sans jouir d'une représentation démocratique aux Communes. Cel argument est d'emblée réfuté par Bentham, hostile à l'Indépendance. Mais al'idée démocratique, par l'intermédiaire de Price, de Prietsley et de bien d'autres, passe, en cet instant précis, d'Amérique en Grande-Bretagne. 5 . Un de ses plus fervents et constants propagateurs en est John Cartwright qui, des 1776, dans Take your Choice, préconise l'introduction du suffrage universel. Mais c'est la Révolution Française qui donne son impulsion décisive au mouvement démocratique en suscitant l'émergence d'un courant de Jacobins anglais rassemblés derrière T. Paine. L'égale participation de tous aux affaires de la cité n'est pas ici seulement requise en tant que chacun doit pouvoir contrôler des décisions susceptibles de l'affecter : elle découle directement d'un statut transcendant, les Droits de l'Homme. L'originalité de la philosophie politique de Bentham apparaît par contraste avec cette pensée.

Les Utilitaristes fondent la démocratie sur l'utilité et donc sur l'intérêt qui ne se conçoit pas nécessairement en termes de droits, mais renvoie davantage à une situation de bonheur mesuré à l'aune du bien-être et de la richesse. D'où l'appréciation de L. Stephen : «Les Jacobins pourraient dénoncer les privilèges

1. Halévy (E.), La formation du radicalisme pbilosophique, vol. 1, La jeunesse de Bentbam, Paris, Félix Alcan, 1901, p. 29.

2. Halévy (E.), L'ésolution de la doctrine utilitaire de 1789 à 1815, op. cit., p. 178.

3. Mill (J.), An essay on government, New York, The Liberal Arts Press, 1955, p. 54.

4. Halévy (E.), L'Evolution de la doctrine utilitaire de 1789 à1815, op. cit., p. 210.

5. Halévy (E.), La formation du radicalisme philosopbique, vol. 1, La jeunesse de Bentham, op. cit., p. 223. 
simplement comme privilèges, et Bentham les dénonce parce qu'ils sont utilisés par les classes privilégiées pour des desseins corrompus [...]. La différence semble être que dans un cas on se prononce au nom de la justice et de l'égalité, dans l'autre cas, au nom de la bienveillance et de l'utilité.". L'existence même de la Déclaration des Droits est condamnable aux yeux de Bentham en tant qu'elle affaiblit l'autorité légale, notamment en bornant son champ d'action. La chose est claire s'agissant de la liberté prise comme droit naturel, car il n'est pas de lois qui ne restreignent la liberté : érigée en droit inaliénable, celle-ci serait une entrave à la législation. Au vrai, l'objection vise l'esprit même de la Déclaration de 1789, «œuvre d'un anarchiste qui foule aux pieds la liberté et la décence [et] nie la validité de la loi [...] convoquant tout le genre humain pour qu'il se soulève en masse et résiste à son exécution " ${ }^{2}$. Ainsi, le souci de l'autorité sociale représentée par la loi et cause du rejet des Droits de l'Homme semble provenir du primat de la société sur l'individu. Au demeurant, c'est de façon explicite que Bentham "objecte que la théorie des Jacobins est trop absolue et trop "individualiste" ${ }_{n}^{3}$. La société représente pour Bentham un corps indifférencié qui prend sens en tant qu'incarnation de l'intérêt général : le seul principe vrai et immuable, c'est l'intérêt général. L'utilité est suprême, qui comprend en soi le droit, la vertu, la vérité et la justice.4. Erigé en norme souveraine, l'intérêt général est appelé à conditionner les droits de l'individu car "C'est par l'intérêt général qu'on découvre les droits de l'hommen's.

La nature et l'étendue des droits individuels sont tributaires de ce qui est l'intérêt général : "c'est en proportion de son caractère avantageux pour la société en question que tel ou tel droit [...] devrait être établi et maintenu, et c'est dans la même proportion de son caractère néfaste qu'il devra être abolin. ${ }^{6}$. Conditionnés par les finalités de la société et mis au service exclusif

1. Stephen (L.), The English utilitarians, vol. 1, Jeremy Bentham, London, Duckworth and Co, 1900 , p. 297. Malgré leurs différences, mais conscients de leur complémentarité, les utilitaristes et les démocrates anglais vont peu à peu se rapprocher pour mener à bien la démocratisation du pays. Une coalition s'étant créée entre Cobbett, Cartwright et Burdett, celui-ci fut élu en 1807 à Westminster, ville qui n'échappera plus aux Radicaux et qui allait en devenir le fief. Or, cette élection donnait l'exemple d'un nouveau style d'organisation électorale, dépendant non pas de la fortune du candidat mais du libre choix des électeurs. (Thompson (E. P.). The making of the english working Class, Harmondsworth, Penguin Books, 1968, p. 509). Ce succès ponctuel cristallise en effet toutes les caractéristiques du radicalisme qui demeure avant tout -un mouvement articulé de protestation, soutenu par une ample désaffection populaire-envers le système. De fait, Cobbett centre essentiellement ses campagnes dans le Register sur l'inadmissible corruption électorale. Qu'il exhorte ainsi les - freebolders, tradesmen ou artisans à se libérer par leur propre volonté du patronage, du soudoiement et de la déférence. (Ibid., p. 508) permet en outre d'établir la sociologie d'un radicalisme qui utilisait surtout sa base populaire comme une masse de manouvre. Son programme, résumé en termes d'attitudes, ssignifie opposition intransigeante au gouvernement; mépris pour la faiblesse des Whigs, opposition aux restrictions mises aux libertés politiques; hostilité ouverte à la corruption et au "système de Pitt" : soutien général à la réforme parlementaire. ( $\left(b_{i} i d\right.$.). Or, l'alliance de ce groupe avec les Benthamites va permettre une plus grande cohérence au plan des idées comme des actions. Bentham, habitant Westminster depuis 1792, adhère définitivement au radicalisme ambiant sous l'influence de J. Mill. Il se lie à Cobbelt, puis, en 1812, rencontre F. Place et, par son intermédiaire, Cartwright. Le groupe développe par voie de presse une propagande démocratique qui s'étend au-delà de Westminster après que fut conclue la paix avec la France. C'est alors qu'à la faveur d'une crise économique qui lui confère un appui populaire, -le radicalisme devient enfin une force populaire. (Halévy (E.), L'évolution de la doctrine utilitaire de 1785 à 1815, op. cit., p. 206). Après bien des années d'oubli, on parle à nouveau de "réforme radicale. en 1816.

2. Parekh (B.), Bentham's political thought, op. cit., p. 263.

3. Stephen (L.), The English utilitarians, vol. I, Jeremy Bentbam, op. cit., p. 294.

4. Halevy (E.), L'Evolution de la doctrine utilitaire, op. cit., p. 48.

5. Ibid.

6. Parekh (B.), Bentham's political thought, op. cit., p. 269. 
de l'utilité générale, les droits individuels ne peuvent être considérés comme des absolus. Leur subordination au primat de la société apparaît dans des termes plus tranchés encore dans le domaine judiciaire puisque Bentham distingue deux niveaux d'action de la loi : l'individu (niveau primaire) et la société (niveau second); et ce aux fins de hiérarchiser les priorités car "le mal causé à un individu peut être finalement moins important que la perte de stabilité dans toute la société. La loi doit donc décourager le mal au niveau second, même si cela semble injuste au niveau primaire.1

C'est la crainte de l'opinion publique qui doit amener le juge à respecter le sentiment du plus grand nombre. Dès lors, s'opère l'identification artificielle des intérêts propres à promouvoir l'intérêt général. Cette conclusion se déduit de l'extrême confiance dans l'influence de l'opinion publique qui caractérise surtout les premiers écrits de Bentham. Ne dit-il pas: "Sans publicité, tous les autres moyens de contrôle sont stériles : comparés à la publicité, tous les autres moyens sont négligeables* 2 ? Bentham souligne les vertus du regards d'autrui à tous les niveaux de la vie sociale. De façon la plus générale qui soit, il considère que toute "opinion" personnelle et donc les "motifs de toutes actions: sont le résultat de "la force de l'opinion publique, appelée aussi "la force de la sanction populaire et morale" ${ }^{3} 3$. Dans la mesure où cette pression est le fait de personnes aux avis le plus souvent divergents, elle reflète le fruit d'une délibération qui conduit Bentham à y voir un véritable "tribunal de l'opinion publique ${ }^{4}$.

Appliquée à l'action politique, cette théorie conduit Bentham, encore en 1830 , semble-t-il à défendre l'institution du vote public car uil est dans la nature de l'homme que l'observation de ses semblables garde éveillé [en lui] un sens du devoir.5. Préfigurant, de façon inattendue, les arguments des opposants du vote secret eux-mêmes, Bentham affirme que cette pratique, comme toute "dissimulation. alimenterait l'hypocrisie et qu'elle ne saurait en aucun cas mettre fin aux corruptions puisqu'il suffirait à un "patron" soucieux de se faire élire, de conditionner la rémunération de ses "clientsn-électeurs au fait qu'il soit élu. Le remède à la non-représentation des classes moyennes consisterait bien davantage pour Bentham à accorder le droit de vote aux contribuables acquittant au moins dix livres d'impôt. Ce document, dont la date est sujette à caution $^{6}$, ne reflète cependant pas la position officielle définitive de Benthan et de ses partisans : en raison même de l'influence exercée par les patrons sur leurs dépendants-électeurs, Bentham reconnut rapidement l'inadéquation du

1. Ginestier (G.), J. Bentham- in Huysman (D.), dir., Dictionnaire des philosophes, Paris, D. Huysman, 1984. C'est ce sacrifice de l'individu au profit du bien commun qui nourrit la critique de l'Utilitarisme chez les théoriciens contemporains, tels que Rawls ou Nozick. Le premier se fonde sur le principe kantien selon lequel nul ne peut traiter un individu comme un moyen, fut-ce en vue de la satisfaction de tous les autres, pour rejeter l'Utilitarisme. Le reproche qu'il lui adresse est en fait de n'être pas assez individualiste. Nozick renchérit en présentant le respect intégral de chaque individu comme une contrainte morale préalable. : à ses yeux, il n'y a pas de poids supérieur des autres par rapport à une seule de nos vies tel qu'il mène à un bien social global supérieur. Il n'y a pas de sacrifice justifié de quelqu'un d'entre nous pour les autres..

2. Cité in Halévy (E.), La formation du radicalisme philosopbique, vol. 3, Le radicalisme philosophique, Paris, Félix Alcan, 1904, p. 169.

3. Dinwiddy (J. R.), dir., -Deontology, together with a table of the springs of action and the article on utilitarianism., The Collected Works of J. Bentham, Oxford, Clarendon Press, 1983, p. 100.

4. Ibid., p. 101.

5. Bentham (J.), A few observations on the ballot, London, Thomas Hatchard, 1830, p. 1.

6. Le tract de Bentham dont on fait ici état est daté de 1830 avec un point d'interrogation par les bibliothécaires du British Museum. 
vote public et la nécessité du ballot. L'opposition initiale - ou occasionnelle - de Bentham au ballot ne doit cependant pas être sous-estimée. Il ne s'agit pas d'une incohérence temporaire. Elle s'inscrit logiquement dans la pensée utilitariste dont la dimension démocratique, un peu laborieusement acquise, valorise volontiers la société au détriment de l'individu (et le ballot protège d'abord le choix individuel). A ce titre, le principe de publicité est érigé en règle d'or. Cette construction théorique est importante parce qu'elle fournira, au terme d'un chassé-croisé progressif, leurs arguments aux opposants du ballot, jusqu'à son adoption en 1872, après que les utilitatistes en soient devenus les principaux partisans, en raison des conditions d'exercice du vote dans l'Angleterre du XIXe siècle.

\section{Le vote sous influence et le ballot-trust comme solution}

Hormis la distorsion bien connue qu'introduisait le poids des bourgs pourris dans la représentation politique, le système électoral anglais se singularisait avant 1832 surtout, mais bien sûr au-delà, par un clientélisme généralisé. Les votes étant émis publiquement, les électeurs étaient soumis aux pressions les plus diverses et surtout de la part de ceux dont ils dépendaient matériellement : «Parfois le patron ou le propriétaire était dans la position d'imposer son choix aux électeurs qui n'étaient qu'une poignée de ses dépendants. Plus souvent, il était obligé de distribuer de l'argent et des faveurs pour garder son emprise sur le bourg et exclure ses rivaux. Certains propriétaires pouvaient donner des ordres; d'autres devaient donner des pots de vin. Certains électeurs montraient une indépendance obstinée. Certains bourgs étaient changeants [fickle] ou indisciplinés [unruly]; d'autres résistaient à toute tentative de contrôle. Les électeurs de ces lieux où le jeu était ouvert avaient un surnom expressif pour leur candidat favori : Mr Most (Monsieur Le Plus (généreux)] ${ }^{1}$. Ces pratiques institutionnalisées incitaient les riches magnats soucieux de se faire élire (ou représenter aux Communes) à acquérir des terres car avec elles il obtenait les voix de ses nouveaux dépendants. Ce genre d'achat n'était toutefois à la portée que d'une minorité de riches industriels et marchands ; si bien qu'à l'indignation morale de plus en plus grande face au dévoiement de l'élection s'ajoutaient les frustrations des classes urbaines riches mais privées de tout rôle politique. Les radicaux se feront aussi les porte-parole de ce sentiment en se prononçant en faveur du vote secret.

Cette revendication fut d'abord promue par J. Bentham qui a donc varié sur cette question, partagé qu'il était entre la règle d'or de la publicité et le souci de permettre à chacun de constituer puis d'exprimer son opinion. Qu'il ait finalement défendu ce dernier objectif est tout à fait conforme au cœur de sa philosophie. Le plus grand bonheur du plus grand nombre que doit promouvoir la démocratie représentative était en effet impossible à atteindre, comme il l'écrit dès 1819, si ule vote létait] l'expression du souhait - non pas de l'électeur, mais de celui par lequel il est soudoyé ou contraint. Tant que cet état de choses demeure, les aspirations, par lesquelles le choix est déterminé, seront - non pas celles du grand nombre [many], gouverné par les intérêts du grand nombre, mais celles de quelques-uns gouvernés par les intérêts d'une minorité. ${ }^{2}$. En 1819, le vote secret semble tellement prioritaire à Bentham,

1. Brock (M.), The great reform Act, London, Hutchinson University Library, 1973, p. 17.

2. Bentham (J.), Radical reform bill - with extracts from the reasons, Londres, E. Wilson-Royal
Exange, 1819, P. 2 . 
qu'il l'inscrit en tête de son projet de réforme parlementaire, explicitement destiné à assurer "une représentation plus adéquate du Peuplen aux Communes. Le ballot est même décrit comme clé de voûte de ce projet : "Sans le secret du suffrage, l'universalité, l'égalité et l'annualité Ides législateurs] seraient pire que rien. ${ }^{1}$. Bentham consacre donc de nombreuses pages de son projet de réforme à exposer des procédures de vote secret très sophistiquées : l'électeur devrait choisir, dans une boîte où il discerne les bulletins à travers un hublot, deux bulletins sur lesquels se trouve inscrit le nom du candidat de son choix avant d'introduire ses avant-bras pour appliquer les deux faces où le nom se trouve inscrit l'une contre l'autre et ne montrer que les faces vierges lorsqu'il donne ses bulletins au "Receiving Clerk qui les enfouit alors dans une urne étanche, en métal. Cette disposition ne vise pas seulement à garantir le secret du vote : elle permet aussi d'isoler l'électeur de la masse des autres électeurs dans laquelle il se trouvait mêlé lorsque le vote était public. "Pour l'organisation du vote, le président du bureau de vote est séparé de la foule, des électeurs et autres, par une barrière ou tout autre séparation efficace [...]. Les estrades où l'on vote sont suffisamment surélevées et séparées de la foule. S'y trouvent 1) le président du bureau de vote ; 2) les assesseurs ; 3) la boîte où l'électeur sélectionne son bulletin ; 4) l'assesseur en charge de l'urne. Pour éviter toute confusion, un corridor sépare les estrades de la foule. Sa largeur doit permettre le passage d'un homme et sa longueur être de six, huit ou dix pieds de manière à former un corridor de taille suffisante avec une barrière à chaque extrémitén 2 . Ce dispositif reflète de façon topographique la dimension individualiste finalement prégnante - de l'utilitarisme. Cette volonté d'isoler l'électeur de la foule participe de la même perspective que le souci de supprimer les relations de clientélisme : le citoyen - dont le type sociologique est en voie de constitution - doit se comporter de façon rationnelle et n'écouter que la voix de ses inclinations et de ses intérêts avant de se prononcer. Seule l'aggrégation de suffrages ainsi réfléchis pourra déboucher sur une représentation fidèle propre à assurer le plus grand bonheur au plus grand nombre.

A ce stade, les Benthamites s'efforcent cependant de concilier cette conception du vote avec le primat de la société sur l'individu qui constitue un principe important de l'utilitarisme : l'électeur ne doit être influencé en rien pour pouvoir se prononcer en conscience, mais, ce faisant, il doit contribuer à promouvoir l'intérêt général ; car le vote n'est pas un droit dont chacun peut faire usage pour défendre ses intérêts, mais un trust dont il est investi par la communauté civique. J. Mill exprime clairement ce point de vue quand il dénonce la contrainte que le dominant peut exercer grâce au vote public : -L'homme riche exerce sur l'électeur les moyens qui sont en son pouvoir pour lui faire commettre un acte criminel au plus haut point - la trahison d'un trust d'une importance inexprimable [...]. [Dans le système du vote public] les gens sont placés dans des circonstances où ils ne peuvent avoir de morale [...], le noble sentiment dans le cœur de chaque homme de regarder l'intérêt public comme le sien propren ${ }^{3}$. Par contraste, le vote secret constitue la clé de voûte de la démocratisation, non seulement parce qu'il permet la représentation du plus grand nombre d'intérêts particuliers, mais aussi parce

1. Ibid., p. 16

2. Ibid., p. 28-29.

3. Mill (J.), On the ballot - from the Westminster review for july 1830, Londres, T. C. Haresard, 1830, p. 10-11. 
que ces intérêts particuliers, lorsqu'ils s'agrègent s'expriment librement, reflètent l'intérêt général.

La conception du vote comme un trust n'est évidemment pas développée par ceux, puisant dans le ajacobinisme» anglais qui y voient un droit ${ }^{2}$. Cette différence indique bien la spécificité et l'ambiguïté des thèses utilitaristes par rapport aux philosophies fondées sur les Droits de l'Homme : ici, le vote est autant un droit qu'un devoir accompli dans la perspective du bien commun. Et si Bentham admet que ce dernier implique le secret du vote, le primat de la société se trouve réaffirmé, de sorte que les opposants au ballot pourront y puiser des arguments. Cette ambiguité reflète aussi les ambitions d'un milieu social en plein essor. Au-delà de ces considérations idéologiques - qui restent premières - la demande du vote secret traduit en effet la volonté de ces classes moyennes d'accéder au pouvoir. Leurs aspirations démocratiques ne sont cependant pas sans mélange puisqu'il s'agit, à bien des égards, à partir du milieu du XIXe siècle surtout, de s'ériger en une nouvelle aristocratie.

\section{Les bases sociologiques du mouvement pour le ballot}

\section{L'ambition des classes moyennes}

L'essor industriel de l'Angleterre s'est accompagné du développement de nouvelles catégories sociales que l'on peut désigner de façon générale du terme de classes moyennes, le pluriel permettant d'en restituer partiellement la diversité : il s'agit aussi bien de riches marchands, d'industriels ou de membres des professions libérales que de groupes bien plus modestes comme les petits commerçants ou les membres de professions libérales les moins prestigieuses. Les plus riches aspiraient surtout à faire reconnaître leur puissance nouvelle dans la sphère politique, notamment en obtenant des députés représentant les villes qu'ils avaient si fortement contribué à développer. Ces hommes, caractérisés par un libéralisme modéré, plaçaient souvent leur confiance dans le parti whig. Ils exprimaient à travers la Edinburgh Review (fondée en 1802) des revendications qui restaient cependant modérées, de peur de n'enflammer les "classes laborieuses" et de n'enclencher un processus révolutionnaire dont la Terreur française les avait détournés à jamais ${ }^{2}$. C.V. Shields explique d'ailleurs "le rôle historique essentiel de l'utilitarisme par son statut de "credo de la classe moyenne britannique ${ }^{3}$. Cette doctrine, en substituant le savoir au statut comme critère d'excellence, semble en effet à même d'assurer la promotion de ce milieu. L'utilitarisme apparaît ici comme une variante du libéralisme. Les Benthamites, en disant que al'autorité ne doit être utilisée que pour promouvoir le plus grand bonheur du plus grand nombre d'individus", suggèrent que ales individus prévoyants, intelligents, industrieux et talentueux doivent être libres de poursuivre leur bonheur sans être entravés par le gouvernementw ${ }^{4}$.

1. Objections to the ballot, answered from the writings and speeches of Mill, Grote, etc., Londres,

H. Hooper, 1837, p. 17.

2. Brock (M.), The great reform act, op. cit., p. 39.

3. Shields (C. V.), -Introduction. à Mill (J.), An essay on government, op. cit., p. 33 .

4. Shields (C. V.), ibid., p. 33. 
Sur le terrain constitutionnel, des radicaux proches de Bentham comme J. Mill ou George Grote se posaient clairement en porte-parole des classes moyennes pour promouvoir la cause des réformes parlementaires. Or, l'un de leurs arguments favoris - la compétence de la classe moyenne - servait directement la demande du vote secret dans la mesure où c'était la meilleure façon d'aattester la compétence. des candidats : a...l'élection d'un candidat, mené par un nombre considérable de votes publics, loin de fournir la présomption de sa compétence, prouve seulement qu'il a soit de nombreux dépendants, soit un nombre inhabituel d'amis privés influents désireux de faire leur sa cause"1. Cette option se trouve développée dans la réponse que les radicaux apportent à l'accusation des conservateurs de vouloir ruiner l'influence légitime de la propriété». Comme le souligne $\mathrm{M}$. Brock, le système d'influence politique qui dominait le vote public était soutenu "par une des plus fortes convictions politiques de l'époque - la doctrine du caractère sacré de la propriété" ${ }^{2}$. Le propriétaire se trouvait auréolé du prestige d'un homme toujours sage doté d'une expérience de la gestion des biens, privés mais aussi publics. Or, de façon significative, les radicaux se défendent de vouloir supprimer l'influence de la propriété par l'institution du vote secret : il s'agit bien plutôt pour eux de substituer un type d'influence à un autre.

J. Mill réfute de bon cœur le qualificatif d'«ennemis de la propriété" dont sont taxés les partisans du vote secret et dénonce les images de spoliation, confiscation, anarchie, bains de sang" qu'on colporte à leur sujet. Il défend la légitimité de l'influence des propriétaires qui, loin d'extorquer un vote sous la menace ou par la corruption, se contentent d'être prévenants et de bon conseil envers leurs employés et dépendants, de manière à s'attirer leur "affection et révérence 3 sans s'immiscer dans leur choix privé. Si J. Mill reconnaît là des qualités de compétence à la classe moyenne, c'est pour l'ériger en nouvelle aristocratie. Au demeurant, J. Mill réinterprète la conception benthamite du vote comme trust dans un sens aristocratique. Il énonce en effet une nouvelle version de la représentation virtuelle en fondant l'exclusion des enfants et des femmes du corps électoral sur le fait que leur intérêt est compris dans celui de leurs parents et maris. Et c'est ce même raisonnement qui peut expliquer l'exclusion du "petit peuple" ; celle-ci, inavouée puisque J. Mill récuse tout cens électoral nécessairement inadéquat, est en effet motivée par le fait que ules opinions de cette classe de gens qui sont au-dessous de la classe moyenne sont formées et leur esprit est dirigé par cette catégorie intelligente et vertueuse qui est le plus immédiatement en contact avec eux [...], à laquelle ils demandent avis et assistance dans toutes leurs nombreuses difficultés, envers laquelle ils éprouvent une dépendance immédiate et quotidienne.... ${ }^{4}$. Ces arguments, par le type de relations sociales qu'ils impliquent, visent, selon Moore, à promouvoir "de nouveaux oligarques 5 . Car les mécanismes de la représentation virtuelle sont ici justifiés en des termes essentiellement aristocratiques de dépendance, d'assistance et d'influence. C'est que, "critiquant leur société, les Benthamites ne critiquaient pas ses valeurs. Ils utilisaient plutôt celles-ci comme un cadre de référence " 6 . A

1. Grote (G.), Essentials of parliamentay reform, Londres, Baldwin and Cradock, 1831, p. 50.

2. Brock (M.). The great reform act, op. cit., p. 36.

3. Mill (J.), On the ballot, op. cit., p. 7.

4. Ibid., p. 90.

5. Moore (D. C.), The politics of deference, Hassoks, The Harvester Press, 1976, p. 417.

6. Ibid., p. 428. 
partir des années 1830 cependant, le ballot tend à apparaître non plus seulement comme la clé de voûte des réformes voulues par les classes moyennes radicales, mais encore comme la revendication permettant leur union avec la "classe laborieuse" (working class).

\section{La logique sociale du débat radical autour du vote secret}

Les radicaux avaient besoin d'un soutien populaire pour espérer voir leurs demandes de réformes aboutir ; parallèlement, ils ne pouvaient adopter une position trop extrême pour ne pas effrayer leurs alliés whigs. La demande du vote secret semble avoir contribué à résoudre ce dilemme dans la mesure où il était l'adjuvant de projets de réforme, par ailleurs modestes, qui garantissait aux "classes laborieuses" une volonté démocratique, celle-ci pouvant s'affaiblir et la demande du ballot disparaître à la demande de certains radicaux après que le soutien populaire eût développé son effet dans la rue. Ces éléments de tactique s'observent tout au long du combat pour le vote secret.

Après l'agitation radicale des années 1817-1824 dominée par le projet de réforme parlementaire de Bentham, les années 1824-1829 sont marquées par une telle indifférence au problème qu'aucune pétition pour la réforme n'est présentée au Parlement au cours de cette période. A l'approche des élections de 1830 , les Whigs, influencés par les idées radicales, s'indignent d'un système électoral dont ils profitent beaucoup moins que les Tories. L'ancienne génération demeure cependant prisonnière de ses appréhensions envers toute réforme qui pourrait dégénérer en révolution. La volonté réformatrice s'affirme, paradoxalement, avec la bénédiction des Tories, après les élections de 1830 qui ont vu l'érosion du pouvoir des notables traditionnels face à la "révolte. de certains de leurs dépendants soucieux de vendre leur vote toujours plus cher. Tories et Whigs se résignent alors à entreprendre une réforme qui consiste essentiellement en une redistribution des sièges en fonction de la population et en une extension de la franchise, à l'exclusion de toute avancée vers le secret du vote. De façon significative, Canning et Peel qui avaient déjà pris conscience de la nécessité d'une réforme dans les années 1820 étaient soucieux d'en rejeter le vote secret, ce qui n'est pas le moindre indice de l'importance de l'enjeu. "Canning et Peel voulaient conserver le vote public de manière à ce que le propriétaire foncier puisse savoir comment votaient ses tenanciers. Ils ne voulaient pas que le vote soit donné à quiconque le réclamait. Ils demandaient un système électoral qui donnerait une grande influence au petit nombre des possédants. Mais ils ne croyaient pas que le système qui avait été acceptable en 1760 puisse être accepté beaucoup plus longtemps* ${ }^{1}$.

Si le regain de popularité des thèses réformistes des années 1829-1830 ne fait pas encore place à la demande du vote secret, c'est dans ce contexte favorable qu'elle va se développer ${ }^{2}$. La remise en cause du niveau du cens était propice à

1. Brock (M.), The great reform act, op. cit., p. 25.

2. Les années 1830-1831 voient en effet fleurir les pamphlets favorables au vote secret. J. T. Barber Beaumont trouve ses bienfaits self-evident (Barber Beaumont (J. T.), One of his Majesty's Justices of the peace for Middlesex and Westminster, thoughts on the causes and cure of the present distresses, with a plan of parliamentary reform, Londres, J. Ridgway, 1830, p. 42) à une époque où une poignée d'hommes en sont partisans : C. Buller - député du Loo Est - y voit une condition nécessaire de la représentation. (Buller (C.), On the necessity of a radical reform, Londres, J. Ridgway, 1831, p. 57) ; H.W. Tancred considère que l'argument le plus puissant en faveur [suite de la note page suivante] 
ce regain d'intérêt pour le vote secret que les deux problèmes étaient effectivement liés, comme le souligne G. Grote : le vote secret "ne peut, sans aucun doute, être rendu efficace si le nombre des électeurs en concurrence pour élire un député est trop petit. Dans ce cas, un homme riche pourrait leur verser des pots de vin en bloc, convenant de payer une certaine somme à chacun après les élections. L'initiative d'une relance du débat dans ces termes revint d'abord à Cobbett et Henry Hunt. Ces deux radicaux produisirent en juillet 1829 un manifeste dans lequel ils demandaient aux "classes laborieuses" de ne souscrire à aucun projet de réforme qui n'incorporerait pas le renouvellement annuel du Parlement, le suffrage universel et le vote secret ${ }^{1}$. Par ces trois demandes, les radicaux faisant office de chefs politiques des classes populaires se prémunissaient contre le risque d'offrir un soutien sans contrepartie aux Whigs.

Juste après avoir formé son gouvernement, Grey - un Whig - chargea Durham - un radical du cabinet - d'animer la réflexion sur la réforme. Il inclut le vote secret dans son projet. Cette demande "était devenue quelque chose comme le cri de bataille radicaln ${ }^{2}$, d'abord parce qu'elle servait leur stratégie et les intérêts des classes moyennes. Les radicaux se démarquaient en effet ainsi des Whigs et se rapprochaient d'autant des "Classes laborieuses" à moindre frais puisque le caractère restreint du suffrage, lui, n'était pas en cause. "Les radicaux avaient besoin de montrer sans risque qu'ils étaient démocrates. Défendre l'idée d'une large diffusion des droits de vote aurait comporté un risque : demander le Ballot n'en comportait aucun. Le Ballot était le moyen d'unir les réformistes de la classe moyenne et ceux des classes laborieuses [working classes]. Il était encore difficile, dans la plupart des endroits, d'amener les deux groupes à collaborer. En incluant le vote secret dans ses propositions, un radical de la classe moyenne pouvait convaincre des réformistes de la classe laborieuse qu'ils pouvaient faire affaire : même s'il ne voulait pas que tous les membres des classes laborieuses aient le droit de vote, il était l'ennemi de la domination artistocratique ${ }^{3}$.

Significativement, le vote secret fut la principale source de conflit au sein du comité chargé d'élaborer le projet de réforme sous la direction de Durham : celui-ci, en bon radical, refusait de céder sur le ballot, malgré l'opposition de Russell (un Whig réformiste qui allait contribuer à bien d'autres projets de réforme au cours du siècle). Le gouvernement de Grey élimina cependant le vote secret du projet de réforme finalement présenté au Parlement en 1832. Celui-ci, qui portait le nombre des électeurs potentiels à 800000 , n'en continuait pas moins à plaire à de nombreux radicaux - ceux qui voyaient surtout dans la mention du vote secret un moyen d'obtenir un soutien populaire à moindre frais - tandis que d'autres, fidèles à leurs principes, préféraient le rejeter en bloc. A peine le projet du gouvernement introduit aux Communes, F. Place, un "Jacobinn, réunit ses troupes à Westminster. Mais alors qu'il faisait voter une résolution demandant le parlement triennal et le vote secret, Joseph Hume, un radical recommandait même au gouvernement de ne

du vote secret est que "la masse des classes respectables du peuple est réputée le désirer" (Tancred (H. W.), A legal review of the origin of the system of representation in England and of its present state with observations on the reform necessary, Londres, H. Butterworth and J. Hatchard, 1831 , p. 120), signe de la propagation de cette idée, au moins dans certains milieux.

1. Brock (M.), The great reform act, op. cit., p. 79.

2. Ibid., p. 140.

3. Ibid. 
pas s'encombrer d'un article sur le vote secret. D'autres, comme W. Cobbett dans son journal le Political Register, faisaient l'éloge du projet tout en regrettant de ne pas y trouver le vote secret. En fait, ou bien les radicaux étaient résignés d'avance et applaudissaient l'octroi d'une influence politique accrue aux classes moyennes que représentait l'abaissement du cens, ou bien ils se consolaient en pensant qu'une assemblée élue selon les nouvelles modalités adopterait, elle, le vote secret sans coup férir ${ }^{1}$. Cette dernière catégorie fut déçue.

Sans doute les élections de 1832 et de 1835 marquèrent-elles l'entrée de nombreux radicaux aux Communes, mais celles de 1837 furent un désastre et ce n'est qu'en raison de sa faible majorité que Melbourne, Premier ministre Whig, accepta - pour conserver l'appui des radicaux - de rouvrir la question du vote secret en 1839 avec la ferme intention de ne rien céder ${ }^{2}$. Désabusés, des fervents partisans du ballot comme Attwood puis Grote démissionnèrent de leur siège. Le coup d'arrêt mis aux réformes et les échecs électoraux des radicaux (plus cinglants encore en 1841), étaient surtout dûs au fait qu'avec la réforme de 1832, la classe moyenne avait obtenu ce qu'elle demandait : "La plupart des nouveaux électeurs voulaient, non pas contester l'aristocratie mais être reconnus par elle : une fois qu'ils avaient acquis la position qui leur revenait légitimement, ils n'étaient pas en faveur de quelque aventure nouvelle. ${ }^{3}$. En particulier, la revendication du vote secret leur apparaissait moins que jamais prioritaire. Sans doute faut-il chercher dans cette évoluton une des origines du mouvement chartiste qui, dès 1837, reprit l'essentiel des demandes radicales, à commencer par le vote secret. L'Association Londonienne des Travailleurs, fondée en 1835 par W. Lovett, milita pour l'extension du droit de suffrage et le développement du système éducatif, en faisant preuve d'une fidélité "plus ou moins consciente aux idées de Bentham" ${ }^{4}$. Lovett prit ensuite l'initiative, avec F. Place, d'une Charte du Peuple présentée comme le nouveau programme radical. De fait, on y demandait le suffrage universel, le scrutin secret, l'égalité des circonscriptions et des élections annuelles, dans la grande tradition benthamite. Une agitation visant à promouvoir ce programme révéla dès lors l'audience de ces thèses dans la population, tout en disqualifiant peu à peu les radicaux. Le mouvement chartiste sera cependant réprimé et le vote secret, comme ses autres demandes, ignoré du gouvernement.

Pendant environ trois décennies, la cause de la réforme - dont celle du ballot - va ainsi se trouver bloquée, en partie parce que les classes moyennes semblaient satisfaites de ce qu'elles avaient obtenu en 1832, mais aussi en raison de la forte opposition que suscitaient surtout l'extension de la franchise et le vote secret. Les arguments avancés par les adversaires du ballot - et leur érosion tardive - demandent, tout comme ceux de ses partisans, à être examinés du point de vue de la cohérence idéologique comme des intérêts sociaux, une double perspective sans laquelle on s'exposerait à un réductionnisme idéaliste ou matérialiste.

1. Ibid., p. 165 .

2. Ibid., p. 317.

3. Ibid., p. 319.

4. Halévy (E.), Histoire du peuple anglais au XIXe siècle, vol. 2, Paris, Hachette, 1973, p. 565. 


\section{Fondements de l'opposition au ballot et circulation des arguments}

Le contexte et les modalités de la revendication du vote secret et de son rejet dans le second tiers du XIXe siècle, de la réforme de 1832 à celle de 1867 , s'apparentent à ceux d'avant 1832 : les Whigs ont le pouvoir avec Russell pour leader et l'appui des Tories contre les radicaux ou, plus souvent des radicaux contre les Tories, voire - c'est même le plus fréquent - contre les Whigs conservateurs menés par Palmerston. Ces radicaux ressemblent à ceux des années 1820-30: ils se recrutent toujours surtout parmi "les magnats de la seconde génération soucieux de jouer un rôle dans la politique et la société proportionnel à la situation éminente qu'ils ont atteint dans l'industrie et dans les villes en généraln'. John Bright et Richard Colden sont les leaders de ce groupe qui a ses origines dans l'Anti-Corn Law League ${ }^{2}$. L'autre pôle du camp radical était constitué de ces hommes de la province qui ont toujours maintenu un "gentry radicalism" 3 aux Communes. Parmi les plus actifs défenseurs de la réforme, on trouve P. Locke King (fils du baron King et député de East Surrey) et F. H. Berkeley, fils illégitime du cinquième comte de Berkeley, député de Bristol et l'avocat obstiné du vote secret. Le premier ayant proposé une extension du suffrage lors de la session de 1851, le second comme le voulait la coutume - introduisit une motion demandant le vote secret en arguant que cela mettrait fin aux pratiques d'intimidation et de corruption des propriétaires fonciers. Joseph Hume renchérit en prédisant que le vote secret contribuerait à transformer la composition de la chambre et à réduire les taxes qui servaient à financer les pots de vin électoraux ${ }^{4}$. Comme par le passé, Tories et Whigs conservateurs firent bloc contre ce projet ; Russell prit cependant l'initiative d'une réforme électorale dont le texte, finalement rejeté sous la pression de Palmerston, ne faisait pas place au vote secret 5 .

Ce processus allait se reproduire en 1854. En 1857, à la suite du succès électoral des Whigs conduits par Palmerston, Disraeli embrasse à son tour la cause des réformes dans l'espoir d'en faire le levier d'une renaissance Tory. Les radicaux, avec Bright, relancèrent l'agitation, surtout dans la perspective d'obtenir une redistribution des sièges plus favorable aux classes moyennes urbaines; ils maintenaient toutefois la demande du vote secret que Berkeley présentait tous les ans. La réforme échoua à nouveau. Les Whigs retrouvèrent le pouvoir avec toutefois un besoin plus grand de l'appoint des radicaux en voix. Les projets de réforme de Russell de 1860 à 1865 n'en suscitèrent pas moins un profond scepticisme dans les rangs radicaux : en 1862 et 1863, Locke, King, Berkeley et Baines, désabusés, ne présentèrent pas l'habituelle motion annuelle en faveur de l'extension de la franchise et du vote secret, persuadés que la collusion des Tories, Peelites et Whigs partisans de Palmerston les

1. Smith (F. B.), The making of the second reform bill, Cambridge, Cambridge University Press, 1966, p. 29.

2. Il n'est pas sans intérêt de donner un aperçu sociologique de ses principaux représentants à la Chambre : W. Scholfield, fils d'un banquier et marchand, député de Birmingham ; J. Stanfeld, fils d'un avoué, brassier et député de Halifax; W. Ewart, fils d'un marchand de Liverpool ; F. Crossley, fils d'un fabriquant de tapis de Halifax; E. Baines, fils du propriétaire du Leeds Mercury ; J. Clay, fils d'un marchand de Londres, tout comme J. Locke; E. A. Leathan, fils d'un banquier quaker de Wakefield (Ibid., p. 29-30).

3. Ibid., p. 30.

4. Ibid., p. 32.

5. Ibid., p. 34. 
mettrait en minorité ${ }^{1}$. Au cours de ces trois décennies, des années 1830 au années 1860 , les tenants et opposants du vote secret s'affrontèrent avec des arguments peu à peu routinisés mais révélateurs de conflits idéologiques plus élaborés que la simple défense d'intérêts sociaux.

\section{Les arguments contre le vote secret}

Les opposants au vote secret recourent à un répertoire d'arguments très large et de niveau très inégal mais, et c'est le plus intéressant, souvent fourni, à l'origine, par les utilitaristes eux-mêmes.

\section{Les précédents étrangers}

Opposants et partisans du vote secret cherchent à tirer parti des exemples étrangers de façon plus ou moins convaincante : H.W. Tancred avance, d'un côté, que le cas américain est positif dans la mesure où une opinion hautement favorable à cette manière de voter prévaut dans ce grand paysn ${ }^{2}$ et E. Beales, Président de la Reform League renchérit 34 ans plus tard en soulignant que le vote secret favorise le déroulement d'élections paisibles comme le prouvent anon seulement son usage dans notre propres colonies là commencer par l'Australiel, en France et en Italie, mais encore par l'exemple magnifique montré par les Etats-Unis. ${ }^{3}$; à l'inverse, Disraeli, dans son discours contre la motion de J. Hume en 1852 tire argument d'une lettre du Gouverneur de l'Etat de New York pour conclure que le vote secret, loin de réduire la corruption électorale aux Etats-Unis, l'a renforcée ${ }^{4}$. On trouve dans ces références aux expériences étrangères une sorte d'invocation quelque peu magique qui repose sur une connaissance très superficielle des réalités.

\section{Corruption et hypocrisie}

Une autre série d'arguments et de contre-arguments à la construction intellectuelle médiocre mérite davantage l'attention dans la mesure où on y décèle le conflit de deux cultures politiques. Les conservateurs objectent de façon récurrente que le vote secret accentuera les risques de corruption. Lord Russell traduit une opinion fort répandue lorsqu'il déclare à la Chambre le 2 juin 1835 : "Je ne peux pas m'empêcher de penser qu'il sera bien plus facile, quand il ne sera plus possible de voir les gens voter et quand vous n'aurez plus le droit de vous enquérir du vote d'un homme, de pratiquer des pots de vin massifs" ${ }^{5}$, parce que chaque électeur pourra empocher en toute impunité les pots de vin venant de différents candidats désormais privés de tout moyen de contrôle. Il faut là toute la patience des radicaux pour expliquer - sans succès - que nul n'aura précisément plus aucun intérêt à pratiquer les pots de vin, étant donné l'incertitude du résultat. De façon plus intéressante, les conservateurs adoptent une posture morale paradoxale mais révélatrice de leur culture politique en critiquant l'hypocrisie que va susciter le vote secret : -La simple apparition du mystère excitera la curiosité ; chaque mot, chaque

\footnotetext{
1. Ibid., p. 47 .

2. Tancred (H. W.), A legal review of the origin of the system of representation in England..., op. cit., p. 123.

3. Speech of Edmond Beales at the meeting at Saint Martin's ball, in support of the [Reform] League, 13 mai 1865 , Londres, J. G. Taylor, 1865 , p. 9.

4. Parliamentary reform. A series of speeches on that subject delivered in the House of Commons by the Right Hon. B. Disraeli (1848-1866), Londres, Longmans, Green and Co, 1867, p. 69.

5. Objections to the ballot, op. cit., p. 10.
} 
regard seront observés, et tôt ou tard la vérité pourra être découverte. En effet, le suffrage de chacun peut généralement être inféré des opinions qu'il développe, si il n'en a aucune; de telle sorte que les gens devront les déguiser aussi assidûment pour éviter cette découverte et même un pur mensonge pourra difficilement être évité. Quelle vie d'hypocrisie serait ainsi organisée ? ${ }^{1}$.

G. Grote répondait à ce genre d'arguments qu'il n'y aurait hypocrisie que si un impétrant entreprenait de questionner un électeur sur son vote, ce qui ne saurait demeurer légitime dans les mœurs politiques à venir et que, de toute façon, ces protestations indignées contre l'hypocrisie ne sauraient être prises au sérieux, provenant de personnes qui la pratiquaient habituellement dans leurs clubs où le vote était secret ${ }^{2}$. Mais la contre-attaque semblait sans pertinence à des propriétaires fonciers qui ne pouvaient comparer les relations entre égaux au sein des clubs et celles qu'ils entretenaient avec leurs dépendants dont ils n'envisageaient même pas qu'ils ne pourraient les interroger. Phillips ne parvient pas à concevoir l'économie d'un système politique où aaucun représentant ne pourrait savoir qui furent ses soutiens [électoraux] sur lesquels il pourrait compter à coup sûr, ou quelles sont leurs vues politiques [...]. L'organisation et la discipline de parti, une nécessité reconnue dans notre constitution représentative, deviendrait donc impraticable; nos gouvernants ne pourraient qu'avec difficulté prendre la mesure de leur force relative au Parlement et l'instabilité continuelle du gouvernement en serait le résultat inévitable ${ }^{3}$.

Ces arguments, comme les précédents d'ailleurs, dissimulent certainement la poursuite, par des dominants, de l'intérêt qu'ils ont à maintenir leur ascendant par le vote public. Mais toute chaîne argumentative stratégique articule un intérêt sur un ensemble de valeurs qui délimitent non seulement le souhaitable pour soi, mais aussi l'horizon du pensable. Quand, le 25 avril 1833, R. Peel réfute aux Communes le bien-fondé du vote secret en demandant : "Pourquoi ne pas offrir aux tenanciers l'occasion de manifester ouvertement leur respect en se conformant eux-mêmes aux opinions on ne peut plus éclairées de leurs propriétaires fonciers ? 4 , il s'emploie autant à défendre ses intérêts qu'il exprime son souci de perpétuer un état d'équilibre (pour lui) en dehors duquel il ne peut concevoir l'ordre politique, faute des catégories adéquates. Phillips lui-même concluait que le vote secret "tendrait à provoquer une révolution sociale et politique étant donné qu'il remettrait en cause des coutumes qui ont longtemps commandé la confiance publique..." 5 . Il était trop impliqué dans le cours des événements pour saisir que l'introduction du vote secret ne serait qu'une des manifestations d'une remise en cause générale des liens sociaux traditionnels dans lesquels s'insérait cette "confiance publique...". Au-delà de ces arguments aux fondements défaillants, ne serait-ce que parce qu'ils trahissent des vues intéressées, les opposants du vote secret vont développer une thèse fort riche qui retournera en quelque sorte les arguments de leurs adversaires contre eux-mêmes, apparemment sans malice.

1. Phillips (H. C. M.), The ballot. Three letters, Londres, Simpkin, Marshall and Co, 1870, p. 5.

2. Grote (G.), The great reform act, op. cit., p. 59.

3. Phillips (H. C. M.), The ballot. Three letters, op. cit., p. 5-6.

4. Objections to the ballot, op. cit., p. 12.

5. Phillips (H. C. M.), The ballot. Three letters, op. cit., p. 16. 
Le ballot est un-british parce qu'opposé au principe de publicité

L'argument le plus fréquent des opposants au ballot repose sur l'idée que le vote secret est un-british dans la mesure où, conformément à une "coutume anglo-saxonne depuis longtemps enracinée" ${ }^{1}$, l'électeur doit avoir le courage, voire la virilité, d'afficher ses opinions. J. Mill avait beau jeu de réfuter cet argument, dès 1830 , en raillant que "parmi les opposants du vote secret au parlement, il y en a dont on ne peut pas dire qu'ils argumentent mais qu'ils gémissent et utilisent des arguments inarticulés. ${ }^{2}$. Cette parade n'est cependant pas suffisante parce qu'au second degré l'argument du vote secret comme un-british reflète un attachement traditionnel à la publicité de la chose politique dont les utilitaristes eux-mêmes furent les premiers théoriciens. Il est ici utile d'anticiper sur le destin du ballot pour examiner les arguments avancés par les députés conservateurs des Communes lors de l'ultime débat parlementaire de 1872. Ces derniers restituent en effet une formulation particulièrement claire de l'association entre tradition britannique et publicité.

Disraeli lui-même considérera alors que le ballot est un-British parce qu'il empêcherait sles classes indépendantes, qui ne sont pas corruptibles et qui ne craignent pas d'être intimidées, de jouir de ce que les Anglais ont toujours honoré - la publicité dans l'exercice de leurs devoirs" :Année après année, ajoute-t-il, nous avons lutté pour rendre la vie politique publique. La publicité est à présent l'âme de notre vie politique. Nous devons au principe de publicité nos principaux bienfaits. Nous avons introduit la publicité dans les affaires du Parlement, dans les tribunaux, dans la presse. Maintenant, on nous demande d'agir en contradiction avec cette évolution que nous suivons depuis si longtemps ${ }^{3}$. Un autre député conservateur, W. H. Smith, s'élève contre la façon dont les Communes s'apprêtent à hypothéquer al'influence vivifiante de l'opinion publique et de la responsabilité publique sur laquelle elles ont toujours tellement mis l'accent" : :Il est toujours apparu d'une suprême importance que l'opinion publique guide, influence et contrôle l'action des électeurs qui devait donc se dérouler au grand journ" ${ }^{4}$. La tradition dont se réclament ces Tories n'est autre que celle ouverte par l'utilitarisme benthamite qui avait bien pour principe (entre autres) la promotion du atribunal de l'opinion publiquen. Cette ironie de l'histoire qui revient, pour les radicaux, à voir leurs pères spirituels fournir à leurs opposants l'essentiel de leur argumentation, s'observe de façon tout aussi nette dans le cas de la conception du vote comme un trust.

\section{Le vote comme trust}

Dans son discours du 3 mai 1865 devant le Parlement, contre le projet de Baine, R. Lowe dénonce comme "sentimentaux" ces "droits abstraits"s au nom desquels certains - dont J. S. Mill dit-il - prétendent faire participer tous les hommes au gouvernement sous prétexte qu'ils sont égaux et y ont un droit

1. Ibid., p. 5 .

2. Mill (J.), On the ballot, op. cit., p. 21.

3. Hansard's parliamentary debates, third series, vol. 212, Londres, C. Buck, 1872 ; débat du 28 juin 1872, col. 354 .

4. Ibid., vol. 211 ; débat du 30 mai 1872, col. 857.

5. The speech of the Rt. Hon. Robert Lowe, upon the second reading of Mr. Baine's Bill for the extension of the bonough rranchise, 3 May 1865, Londres, Bickers, [s.d.], p. 4. 
inaliénable. Il a alors beau jeu de citer les Principles of Morals and Legislation de Bentham ("l'avocat du suffrage universel", souligne-t-il), celui-ci ayant en effet toujours rejeté ce discours métaphysique sur les droits de l'homme au profit d'une attention aux conditions pratiques du bonheur du plus grand nombre. Lors du même débat, Horseman (partisan de Disraeli) enfonce le clou en affirmant que le droit de vote eest conféré par la loi, non pas comme la matière d'un droit privé mais comme un trust public ' ${ }^{1}$. Lord Palmerston, un Whig conservateur, avait comme institutionnalisé cette conception un an auparavant dans un discours prononcé pour contrer la motion rituelle de Berkeley en faveur du ballot : "Je m'oppose à cette motion parce qu'elle repose sur un faux postulat [...]. [Berkeley] considère le droit de vote comme un droit personnel alors que je pars du principe qu'il s'agit d'un trust devant être exercé au nom de la communauté dans son ensemblen ${ }^{2}$. Dès lors que le vote n'appartient pas à la minorité des citoyens auxquels ce droit est reconnu mais que cette minorité est mandatée par le grand nombre en vertu de ses compétences, cette minorité doit l'exercer publiquement, de manière à ne pas pouvoir trahir la confiance de la majorité. Il lui faut voter sous le regard de cette dernière. Cette argumentation s'inspire directement de l'utilitarisme benthamite et constitue la principale ligne de défense de Whigs tels que Palmerston, mais aussi Gladstone, et bien sûr de conservateurs comme Atkinson qui expliquait ainsi en 1858 que le vote est une chose relative et non une chose absolue, car il procède des relations d'intérêt qui portent sur toute la population. : sachant qu'une minorité a la possibilité de voter, le vote doit être public de manière à ce que le reste de la population - concerné par les conséquences de ce vote - exerce une pression morale sur les électeurs ${ }^{3}$. Cet accent mis sur les vertus du contrôle populaire sur un acte qui est un trust dans la mesure où il engage toute la société renvoie aux positions de Bentham sur le tribunal de l'opinion publique ${ }^{4}$.

L'argument reprenant l'idée du vote comme trust et les conséquences qui en découlaient au regard de son caractère nécessairement public fut cependant

1. Reform, The debate on Mr Baine's bill in the House of Commons on May 3rd and May 8th 1865, Londres, Bradbury and Evans, 1865, p. 67.

2. Cité dans Hansard's parliamentary debates, vol. 209, col. 1176.

3. Atkinson (W.), The franchise and voting by ballot considered and explained, Londres, Longman, Brown, Green, Longmans and Roberts, 1858, p. 60.

4. D'ailleurs Atkinson tire argument de la défense par les Benthamites du vote public à l'assemblée pour demander l'application de la même procédure lors des élections générales : "Ils déclarent que dans ce cas [où le vote serait secret au Parlement], il y aurait une tendance à sacrifier les intérêts du peuple en général. Je maintiens qu'il est juste que le peuple doive connaître la manière suivant laquelle ses représentants votent. La fonction à laquelle les représentants sont élus est une fonction de haute confiance [ $b i g b$ trusti), et étant telle, chaque exécution du trust doit être conduite de la manière la plus publique, sans peur et honorable [...]. Je maintiens que le cas des électeurs et celui des représentants, où le devoir élevé qu'ils sont appelés à réaliser étant un public trust sont analogues et que, dans ces conditions, un seul principe de vote est applicable aux deux. (Atkinson (W.), The franchise and woting by ballot, op. cit., p. 59-60). Il est tout à fait exact que les radicaux ont toujours demandé la publicité des votes au Parlement, une .Charte. de 1831 exigeant même, pour que le contrôle soit effectif, que -les sessions de chaque corps représentatif soient tenues dans un bấtiment où ses délibérations puissent être observées par 500 personnes au moins qui pourront être admises sans droit d'entrée ou charge. (The New Charter bumbly addressed to the King and both Houses of Parliament, proposed as the basis of a Constitution for the Government of Great-Britain and Ireland and as a sustitute for the reform bill rejected by the Lords, Londres, W. Strange, 1831, p. 14). Pour les Radicaux, il existe toutefois une différence évidente entre l'élection des représentants et les votes de ceux-ci au Parlement : pour les Benthamites, les seconds sont libres de toute pression, à la différence de la première, pour les .jacobins., le vote des députés est un droit personnel et, pour ces deux groupes, la priorité est le contrôle des législateurs et gouvernants de manière à identifier leur intérêt (notamment synonyme de réélection) et ceux de leurs mandants. 
celui qui hypothéqua le plus clairement l'adoption du ballot parce qu'il constituait un terrain d'entente pour les Whigs comme pour les Tories. De nombreux radicaux contestèrent naturellement cette interprétation et s'attachèrent à accréditer l'idée selon laquelle le vote constituait un droit : Beales considère que l'idée d'un tel trust est répugnante au sens commun et inconnue de la loi $[$...]. Comment un non-électeur peut-il obliger un électeur à voter comme lui, le non-électeur, souhaiterait le faire ou à rendre compte du vote donné ou de la façon dont il a exercé son trust ?w1. Pourtant, cette assimilation d'un vote à un trust demeurait fortement enracinée et c'est en s'y référant que Disraeli put réfuter une tentative de réforme comme celle de 1852 : comment justifier, demande-t-il, l'alliance du suffrage restreint et du vote secret qui permettrait à une minorité de se soustraire au "contrôle des millions d'hommes non-habilités à voter ?" ; cela reviendrait à établir une "oligarchie des plus oppressives" ${ }^{2}$. Le vote secret, souligne-t-il, n'est légitime qu'à partir du moment où il est couplé avec le suffrage universel. Or accepter celui-ci est hors de propos dans la mesure où cela reviendrait à accepter la souveraineté du peuple et donc à renier le fondement de la Constitution anglaise car ala Constitution de l'Angleterre c'est la souveraineté de la Reine Victoria ${ }^{3}$.

C'est effectivement l'évolution de l'Angleterre vers le suffrage universel et le déclin corrélatif de la thèse du vote comme trust qui va conduire Gladstone à se prononcer pour le ballot dont il était jusqu'alors l'adversaire. La crainte de l'irruption des masses sur la scène politique, liée précisément à l'extension du droit de vote, sera aussi à l'origine de la conversion de certains Whigs au ballot mais amènera un radical comme J. S. Mill à remettre en cause le bien fondé du vote secret. Ce chassé-croisé domine le débat sur le vote secret dans les années 1868-1872, date d'adoption du projet de loi instituant le vote secret.

\section{Renouvellement et circulation des arguments}

Les inquiétudes d'un utilitariste atypique : J. S. Mill

En réponse au projet de réforme à venir du gouvernement de Lord Aberdeen, J. S. Mill exprima en 1854 des Thoughts on the Parliamentary Reforms où il remettait en cause son soutien au vote secret. De son point de vue, cette mesure n'était plus d'actualité dans la mesure où, hormis les quelques bourgs les plus petits ${ }^{4}$, les circonscriptions où les dominants exerçaient une influence sur les électeurs étaient devenus rares. Le danger était désormais ailleurs : "A présent, je considère qu'une source de maux bien plus grands est l'égoïsme, ou la partialité égö̈ste de l'électeur lui-même. Un vote "vil et malfaisant" est maintenant, j'en suis convaincu, plus souvent celui inspiré par les intérêts personnels de l'électeur ou par des intérêts de classe ou par les tendances mesquines de son propre esprit, que par la peur de représailles éventuelles de la part d'autrui ; et le vote secret lui [à l'électeur] permettra de céder à ces mauvais penchants libre de toute honte ou responsabiliténs.

1. Speech of Edmond Beales..., op. cit., p. 6.

2. Parliamentary reform..., op. cit., p. 67.

3. Ibid., P. 68.

4. Mill (J. S.), Thoughts on the parliamentary reform, Londres, J.W. Parker and Sons, 1859, p. 40.

5. Ibid., p. 38-39. 
Cette argumentation sera développée par J. S. Mill dans Le gouvernement représentatif quelques années plus tard. Refusant au vote la qualité de "droit", pour en faire un devoir dont les retombées impliquent toute la communauté, J. $S$. Mill avance que l'électeur doit se conformer à "ce que la société semble attendre de lui ${ }^{1}$ : c'est pourquoi il faut que "le devoir de voter, comme tout autre devoir public, soit accompli devant les yeux du public" ${ }^{2}$. Car si "le pouvoir de contraindre les votants a décliné ${ }^{3}$, ceux-ci risquent à présent de se prononcer en faveur de "l'intérêt personnel ou de l'intérêt de classe", chose qu'ils n'oseront pas sous le regard des autres. Cette argumentation a des accents benthamites très clairs qui la situe dans le droit fil des thèses sur "le tribunal de l'opinion publique.. Cette problématique se trouve toutefois renouvelée par l'évolution du contexte social : ce n'est plus l'inféodation aux dominants qui est désormais redoutée mais l'égoïsme et surtout l'aliénation de sa volonté à la classe sociale. Ce thème qui prendra une place croissante dans l'œuvre de J. S. Mill est à relier à sa vision globale de la société politique. Notamment sous l'influence de Tocqueville et de ses descriptions de la démocratie américaine, J. S. Mill a peu à peu conçu une aversion pour tout ce qui évoque le pouvoir des masses (de l'uniformisation des mœurs sous l'effet de la tyrannie du grand nombre jusqu'à l'obsession de l'égalité) comme en témoigne surtout son livre On liberty. Ces vues amènent $\mathrm{J}$. S. Mill à contester toute remise en cause de la théorie du vote comme trust qui commande son caractère public; en effet, il ne croit plus souhaitable l'extension du suffrage qui l'avait conduit à militer pour le vote secret : "L'opinion et les souhaits des classes de travailleurs les plus pauvres et les plus frustres peuvent être utiles en tant qu'une influence parmi d'autres sur l'esprit des électeurs comme sur l'assemblée; mais il serait hautement nuisible de leur donner une influence prépondérante en les admettant, dans l'état actuel de leur morale et de leur intelligence, à exercer pleinement le vote. C'est précisément cette influence indirecte de ceux qui n'ont pas le droit de vote sur ceux qui l'ont qui, par son développement progressif, adoucit la transition vers toute nouvelle extension de la franchise et est le moyen par lequel, lorsque les choses sont mûres, l'extension s'opère paisiblement. ${ }^{4}$. Hormis une certaine peur des masses typique d'un système de pensée fort élitiste, mais aussi soucieux de réserver le vote à ceux considérés comme compétents - on lit ici une conception du vote comme un trust 5 .

Certains radicaux qui considéraient J. S. Mill comme un traître fournissant des arguments aux conservateurs s'efforcèrent de lui répondre. Un auteur anonyme se présentant comme un électeur de Westminster (circonscription de J. S. Mill) souligne combien le cadre dans lequel J. S. Mill s'est fait élire est atypique : partout ailleurs, on observe une pérennité de l'influence des dominants et un

\footnotetext{
1. Mill (J. S.), Le gouvernement représentatif, Paris, Guillaumin, 1865, p. 228.

2. Ibid., p. 229.

3. Ibid., p. 231.

4. Ibid., p. $42-43$.

5. Cette conception est peut-être la clé de la démocratisation à l'anglaise; le vote public semble en effet avoir doublement contribué à l'élargissement progressif du droit de suffrage : d'une part, il était une garantie pour les conservateurs qui, sûrs de perpétuer leur influence, pouvaient plus facilement concéder l'extension de la franchise comme on l'a $\mathrm{vu}$, d'autre part, la pression que les non-électeurs étaient censés exercer sur les électeurs grâce au caractère public du vote était perçue par les Radicaux modérés et les Whigs progressistes comme une phase d'initiation - d'éducation comme ils auraient dit - à la politique. Que la politisation des masses ait effectivement progressé par ce biais semble quelque peu hypothétique. Toujours est-il que Gladstone pourra le penser, à la différence de J.S. Mill et en déduire la nécessité d'étendre le droit de suffrage d'oú découlera l'institution du vote secret.
} 
développement de la corruption ${ }^{1}$. Surtout, ce critique de J. S. Mill s'emploie à trouver une parade à ses arguments en démontrant que le secret doit s'appliquer au vote, qu'il soit perçu comme un droit ou comme un trust: "Le vote est soit un droit, soit un trust; si c'est un droit, cela implique sans aucun doute un devoir en contrepartie - le devoir d'exercer ce droit pour le bien public. La tâche, cependant, de juger comment exercer ce droit de vote appartient à l'électeur individuel, influencé par aucune autre considération que celles de la raison et de la justice [...]. Dans le dernier cas Idu vote comme trust], la communauté dit, en effet, nous vous avons investi [entrusted] de la faculté de voter ; vous devez en user suivant votre conscience; voici nos motivations pour telle ou telle prise de position; jugez, décidez et agissez suivant les convictions qu'elles produisent. La question de savoir si l'électeur a une tendance à agir correctement ou non n'est pas pertinente par rapport au problème du vote secret qui vise à assurer la liberté de choix ${ }^{2}$. Cet auteur a visiblement déplacé le débat sur un terrain cher aux radicaux les plus influencés par l'idéologie des droits de l'homme : le fondement de leur raisonnement demeure l'inaliénable capacité qui doit être reconnue à l'homme d'exprimer sa préférence politique. Or, ces postulats sont en grande partie étrangers aux auteurs britanniques, à commencer par Bentham et ce n'est pas sous leur ascendant exclusif que Gladstone évoluera vers le vote secret, signe que malgré la permanence d'influence extérieure (celle des idéaux français et révolutionnaires), l'Angleterre a accompli les divers aspects de sa démocratisation suivant un itinéraire original mêlant deux courants de philosophie politique (anglo-saxon et français).

\section{La conversion raisonnée de Gladstone au ballot}

Les années 1860 représentent une phase de maturation intellectuelle décisive dans le parcours politique de B. Gladstone. En 1859, il votait encore contre l'extension du suffrage et le ballot ${ }^{3}$. Mais dès 1860 , "bien que ses vues sur le mécanisme de la Réforme demeuraient presqu'inchangées, son sens du devoir moral envers les membres des classes laborieuses commencèrent à le travailler et il se persuada peu à peu qu'il devait intégrer les plus honnêtes d'entre eux dans la nation politique ${ }^{4}$. A côté de cette conviction - qui renvoie à l'idée des Droits de l'Homme selon laquelle l'égalité fondamentale de tous les autorise à prétendre à un traitement respectueux de leur dignité on retrouve des conceptions bien anglaises dans le réformisme de Gladstone, surtout pour ce qui concerne le vote secret.

Lors de la présentation du projet de loi qui devait étendre le corps électoral de façon significative en 1866, Gladstone spécifia qu'il n'y inclurait pas la question du vote secret à dessein, parce qu'il n'y était pas favorable 5 . Trois ans plus tard, il se déclara partisan du ballot. Entre-temps, les élections de 1868 avaient été marquées par deux phénomènes à ses yeux déterminants, le développement de la corruption et la remise en cause des fondements du vote

1. A Westminster elector. Mr. J.S. Mill and the ballot : a criticism of bis opinion as expressed in -Thoughts on Parliamentary Reform*, Londres, W. Ridgway, 1869, p. 4-6.

2. Ibid., p. 5.

3. Smith (F. B.), The making of the second reform bill, op. cit., p. 48.

4. Ibid.

5. O'Leary (C.), The elimination of corrupt practices in British elections 1868-1911, Oxford, Clarendon Press, 1962, p. 58. Je remercie Alain Garrigou de m'avoir communiqué cette référence dont le chapitre 3 a fourni la matière des paragraphes qui suivent. 
comme trust. Dès après le scrutin, le gouvernement de Gladstone nomma un Select Committee chargé d'enquêter sur les dépenses, sans précédent, observées à cette occasion. Le Comité, dans son rapport, attribua ces dernières à l'achat massif des voix, proportionnel à l'extension du corps électoral. Il révéla aussi des cas de représailles de la part des propriétaires fonciers qui, mécontents du vote émis par leurs tenanciers, s'en étaient débarrassés ${ }^{1}$. Pour remédier à toutes ces influences extérieures, le Comité recommanda l'adoption du vote secret en s'inspirant notamment des techniques utilisées par deux Etats d'Australie (Victoria et South Australia) depuis 1856 pour les élections locales.

Dès avant la remise de ce rapport, un député radical, E. A. Leatham, introduisit un projet de loi en faveur du ballot en mars 1870. Gladstone se déclara pour la première fois d'accord avec le principe d'un vote secret au nom d'une logique qui n'empruntait pas ses arguments à l'idéalisme des Droits de l'Homme : jusqu'à présent, il était resté fidèle à l'idée du vote comme trust parce que seule une minorité avait le droit de vote et devait donc l'exercer sous le contrôle du grand nombre ; à présent que le suffrage était élargi, le caractère public du vote ne se justifiait plus. Disraeli s'éleva contre ce raisonnement de fait un peu contradictoire. Newdegate, un député conservateur aux Communes, se faisait le porte-parole lorsqu'il objectait que : "même si la franchise a été étendue et même si nous parvenions au suffrage universel et que tout homme ayant atteint l'âge de la sagesse puisse voter, le vote n'en resterait pas moins un trust parce qu'il y aurait encore une grande partie de la communauté, les femmes et les mineurs, soumis aux lois, à la fiscalité, etc., dont les intérêts seraient en charge de ceux disposant du droit de vote "2 Néanmoins, bien des conservateurs semblent avoir souscrit au raisonnement de Gladstone ${ }^{3}$.

Désormais, Gladstone considère le vote secret comme un droit. Il faut cependant souligner qu'il est parvenu à cette conclusion au terme d'un cheminement intellectuel qui, conformément à la voie ouverte par l'utilitarisme, emprunta très peu à l'aidéalisme» de la Révolution Française et à ses valeurs individualistes. En outre, bien des libéraux invoquaient non pas l'argument du droit, mais celui, développé par J. S. Mill, de l'influence des classes sociales, comme en témoigne le débat ultime de 1872. Ce dernier débat parlementaire mérite une attention particulière car on $y$ trouve rassemblés les arguments échangés depuis cinquante ans et sa vigueur, tout comme son caractère interminable, témoignent de l'enjeu - peut-être supérieur à celui de l'extension du suffrage - que représentait le secret du vote.

\section{L'ultime débat}

Le débat conduisant à l'adoption du ballot par le Parlement s'étala sur deux ans, trois projets de loi ayant dû être successivement présentés par le gouvernement. Le premier, en 1871, avorta en grande partie en raison du manque de détermination du gouvernement; le second, la même année, suscita de très nombreux amendements, tant du côté libéral que conservateur.

1. A. Garrigou mentionne des cas comparables (-Le secret de l'isoloir", art. cit., p. 28).

2. Hansard's parliamentary debates, op. cit., vol. 209 ; débat du 29 février 1872, col. 1176.

3. The Gladstone administration - from the year 1869 to the close of the session of 1872, Westminster, The National Union of Conservative and Constitutional Associations, 1872, p. 14. 
Les députés irlandais des deux camps s'inquiétaient de la façon dont le ballot renforcerait l'influence de l'Eglise catholique sur les électeurs et du tout nouveau Home Rule party: à leurs yeux, voter en secret permettrait à l'électeur irlandais de suivre sans retenue son penchant nationaliste. Le leader de l'opposition, Cross, multiplia ensuite les obstructions en invoquant tous les arguments disponibles, de la pertinence du vote comme trust au déclin des influences illegitimes. De sorte que le projet de loi fut transmis avec retard à la Chambre des Lords qui refusa de l'examiner faute de temps ${ }^{1}$. Ces débats de 1.871 ont fait l'objet d'un compte rendu fidele de O'Leary, tandis que celui de 1872 ne semble pas avoir retenu l'attention qu'il méritait. Certains arguments s'y renouvellent en effet de façon inattendue ou tout du moins intéressante.

Les arguments des partisans du ballot : la peur des "classes laborieuses" à la Chambre Haute

Tout comme en 1871 , le projet de loi est présenté et défendu à la Chambre des Communes par un des ministres radicaux du gouvernement de Gladstone, W. E. Forster. Celui-ci ne fait pas preuve d'une grande originalité lorsqu'il motive son projet en vertu du vœu de s'énorme majorité des électeurs" d'être "accessibles à la persuasion mais pas exposés à la corcition" ${ }^{2}$. Son projet ayant été adopté sans surprise le 30 mai 1872, par 274 voix contre 216, il fut transmis à la Chambre des Lords où le débat rebondit. Lord Ripon, chargé de piloter le projet dans cette assemblée, utilisa un argument nouveau fraîchement introduit par Gladstone lui-même et selon lequel le vote secret devait protéger les électeurs contre la pression des syndicats qui auraient récemment demandé à voir les bulletins lors d'une élection à Sheffield ${ }^{3}$ : "On dit que les influences sur lesquelles repose l'intimidation d'électeurs individuels, comme celles des propriétaires fonciers, des employeurs et autres, ont décru et doivent continuer à décroître; mais je me réfère à un autre système d'intimidation, qui est très susceptible de prospérer dans le cadre d'un droit de suffrage élargi - l'intimidation par des corps tels que les syndicats ${ }^{4}$. Lord Ripon, souscrivant à la crainte exprimée par J. S. Mill de voir les "masses" dominées par un sentiment de classe, en déduit donc une conclusion inverse : il ne rejette pas le ballot parce qu'il permettrait aux égö̈smes corporatistes de s'exprimer dans le secret de l'isoloir, au contraire, il y voit un moyen de soustraire l'individu à l'influence des institutions ouvrières en plein essor comme les syndicats.

Des opposants du ballot, comme le Whig conservateur Lord Frey, avaient tiré argument des critiques du vote secret par J. S. Mill5. Certains ne se montrèrent d'ailleurs pas convaincus par Lord Ripon, comme l'indiqua un député conservateur (Stafford Northcote) aux Communes : "Si les syndicats se mettent en tête qu'il est important pour eux d'interférer avec le processus électoral et s'ils sont déterminés à exercer cœrcition et intimidation sur leurs membres pour les amener à voter dans un sens particulier, ils seront en mesure de contraindre et d'intimider dans le cadre du vote secret tout comme dans celui

1. O'Leary (C.), The elimination of corrupt practices in British elections 1868-1911, op. cil., p. 79.

2. Hansard's parliamentary debates, third series, vol. 211, Londres, Cornelius Buck, 1872 ; débat du 30 mai 1872, col. 880 .

3. Ibid., vol. 210 ; débat du 18 avril 1872 , col. 1506 .

4. Ibid., débat du 10 juin 1872, col. 1425. A. Garrigou signale que les syndicats figuraient aussi parmi les instirutions visées par l'isoloir en France ("Le secret de l'isoloir", art. cit., p. 28).

5. Ibid., débat du 10 juin 1872 , col. 1435. 
du vote public [...]. Une organisation d'hommes ayant toujours affaire à ses membres, les voyant tous les jours, disposant d'un système d'espionnage permanent, d'un système permanent d'interrogatoires et de vérifications, serait en mesure, tôt ou tard, en recourant à une espèce de tortures morales, de découvrir comment ses membres ont votém. Cette réfutation de l'argument exprimé par Lord Ripon - et par d'autres avant lui - est cependant révélatrice de l'ampleur des craintes suscitées au sein de l'élite conservatrice par la constitution des "classes laborieuses" en force politique et Stafford Northcote reconnaît d'ailleurs que al'inquiétude suscitée par les syndicats a sans aucun doute converti au vote secret certains de ces opposants d'hier. Les arguments des Lords hostiles au ballot reflètent d'ailleurs surtout leur réticence à entrer dans une "société de masse".

\section{Dernière crispation de l'aristocratie}

Les principaux Lords menant le combat contre le ballot, en 1872 comme en 1871, le duc de Richmond et le comte de Shaftesbury, révèlent dans leur discours un système de valeurs cohérent, fondamentalement aristocratique et, à ce titre, incompatible avec le vote secret. Leur chaîne argumentaire peut se résumer ainsi : le ballot est un-British parce que la tradition britannique repose sur la plus grande publicité possible en vertu non pas seulement - ni même d'abord - du rôle bénéfique de l'opinion publique mais d'un code d'honneur qui implique que l'électeur ait le courage d'afficher son vote; par contraste, le vote secret introduit l'hypocrisie dans le vie publique, sape la confiance des relations sociales traditionnelles, dont la structure verticale est revendiquée parce qu'au fond c'est la marche du monde moderne vers l'égalité qui est rejetée. Cette reconstitution de discours plus ou moins explicite peut être aisément démontrée à partir d'extraits des débats de 1872 provenant surtout des propos de Richmond et Shaftesbury, mais d'autres Lords aussi.

- Le ballot est un-British parce qu'il ignore le principe traditionnel de publicité : "Je considère qu'il [le ballot] est contraire aux vieux principes anglais que nous suivons depuis longtemps selon lesquels le devoir public doit être accompli en pleine lumière, dans la clarté ardente de la lumière publique et avec l'idée que vos concitoyens sont concernés par vos actes" ${ }^{2}$ (le comte de Carnarvon).

- Le vote public ne permet pas seulement à l'opinion publique d'exercer son influence, il est aussi typiquement anglais parce qu'il permet de manifester du courage : secret, le vote perd cette noblesse : "Ce projet de loi contribuera grandement à annihiler dans le pays ce courage politique qu'il devrait être de notre devoir de favoriser" ${ }^{3}$; "Pourquoi la grande majorité des gens de ce pays, qui tire de la fierté à voter de façon ouverte, serait-elle obligée de satisfaire le caprice d'une petite minorité en votant comme l'indique ce projet de loi ? ${ }^{4}$ (duc de Richmond).

- En remettant en cause un système électoral fondé sur des valeurs de courage et de façon générale sur un code moral traditionnel, le ballot sape les bases des relations sociales : le vote secret "force l'homme, tel un animal

1. Ibid., débat du 30 mai 1872 , col. $877-878$.

2. Ibid., débat du 10 juin 1872 , col. 1464 .

3. Ibid., débat du 30 mai 1872 , col. 878 .

4. Ibid., débat du 17 juin 1872, col. 1814 . 
rampant, à se comporter sournoisement, la queue entre les jambes; et juste au moment où les hommes s'éveillent au sens de leur dignité, vous insistez pour qu'ils ne déclarent pas leurs sentiments et qu'ils ne s'acquittent pas de leur devoir sous le regard de toute la communauté [...]. Nombre d'hommes vont passer leur vie dans la suspicion, car les plus honnêtes ne pourront jamais prouver qu'ils ont voté comme ils le disent et vous allez détourner du vote les meilleurs électeur's qui préférerons perdre ce droit plutôt que de faire l'objet du doute et de fausses représentations." (comte de Shaftesbury). Le duc de Richmond déclara aussi sur ce thème : "Le projet de loi semble perturber les relations qui ont jusqu'ici prévalu et qui doivent prévaloir, entre les électeurs et les élus $x^{2}$.

- Les relations en question, et les rapports sociaux en général, sont d'ordre hiérarchique et la réaction suscitée par le ballot procède précisément de la menace qu'il fait peser sur cet agencement : :Si le vote secret est introduit, des agitateurs passeront à travers le pays, de maison en maison, pour persuader les gens de voter pour tels ou tels candidats en disant que s'ils entrent au Parlement, non seulement les impôts seront diminués - un argument somme toute légitime - mais aussi qu'au moyen de quelque petite loi il pourrait y avoir une répartition meilleure et plus juste de la propriété. Ils sont nombreux dans cette classe, des hommes religieux par-dessus le marché, à considérer que certains sont trop riches et d'autres trop pauvres; et bien qu'ils répugneront à user de moyens violents, ils n'auront aucun scrupule à rétablir l'équilibre [social] par la voie législative. Et pourtant, ils ne l'avoueront jamais lors d'un vote public"s (comte de Shaftesbury).

Le vote secret est donc finalement redouté par les Lords parce qu'il annule le contrôle des dominants locaux sous lequel les électeurs n'osent pas encore remettre en cause les inégalités. Soucieux de préserver l'essentiel, les Lords retournèrent aux Communes un projet de loi assorti de nombreux amendements dont trois visaient à atténuer la rigueur du secret : le vote secret serait optionnel pour permettre aux hommes de cœur de voter en public, selon les termes du duc de Richmond, l'auteur des principaux amendements ${ }^{4}$; les bulletins seraient numérotés, au moment du vote, de manière à pouvoir retrouver les électeurs les ayant émis, lors d'une enquête que une éventuelle fraude ; le vote secret n'était acquis que jusqu'en 1880, date à laquelle la question serait réexaminée. L'importance des restrictions apportées au projet de loi par les Lords mécontenta Gladstone, déjà irrité par la durée d'un débat engagé au début de $1871^{5}$. Pourtant, le gouvernement, et surtout Forster, en charge du dossier aux Communes, fit preuve d'une attitude conciliante.

Une loi incomplète?

Forster concéda tout d'abord la nécessité de pouvoir retrouver l'auteur d'un vote lors d'une enquête en cas de fraude électorale, qu'une personne soit soupçonnée d'avoir voté plusieurs fois ou qu'elle ait émis un vote sans satisfaire aux critères conditionnant ce droit. L'amendement proposé par Forster stipulait que chaque bulletin devait comporter au dos un numéro de

1. Ibid., débat du 10 juin 1872 , col. 1456-1457.

2. Ibid., débat du 10 juin 1872 , col. 1449 .

3. Ibid., débat du 10 juin 1872 , col. 1455-1456.

4. Ibid., débat du 10 juin 1872, col. 1444 .

5. Ibid., vol. 212 , débat du ler juillet 1872, col. 476-477. 
série qui figurerait aussi sur la contremarque signée par l'électeur ${ }^{1}$. Cette clause était lourde de conséquence car elle pouvait faire douter l'électeur des garanties de secret entourant son vote, et donc perpétuer l'influence des dominants locaux.

La question du vote secret optionnel recoupait celle posée par l'analphabétisme des électeurs depuis l'élargissement du droit de suffrage. Ce problème avait été considéré dès les premiers débats de 1872 et réglé, à ce moment, d'une façon peu satisfaisante : Forster, interpellé à ce sujet, avait produit un amendement aux termes duquel les électeurs qui prouveraient leur incapacité à lire le bulletin de vote au moyen d'un certificat délivré par un magistrat pourrait demander au président du bureau de vote de remplir le bulletin pour eux ${ }^{2}$. Cette disposition comportait de nombreux défauts. Bien des électeurs illettrés ne seraient pas en mesure - faute de "compétence" ou de temps - d'effectuer ce genre de démarche préalable au vote auprès d'un magistrat. Par ailleurs, le secret du vote se trouvait mis en cause par l'intervention du président du bureau de vote qui était entouré d'assesseurs représentant les différents candidats. Forster reconnaissait qu'il s'agissait là d'une faille grave du projet, mais qu'il avait dû la concéder sous les pressions ${ }^{3}$. L'amendement de Forster est d'autant plus étonnant qu'il avait connaissance de procédures permettant de tourner la difficulté : en Australie, le recours aux chiffres ou à des couleurs était déjà en usage pour identifier les candidats ${ }^{4}$. Les Lords hostiles au ballot trouveront dans ces imperfections du projet un argument en faveur du "vote secret optionnel" puisque certains, les analphabètes, en bénéficiaient déjà. Surtout, ils proposeront d'amender le texte de Forster en remplaçant l'obligation d'un certificat signé de la main d'un magistrat par une une simple déclaration de l'électeur au président du bureau de vote. Tout électeur de basse extraction aurait alors pu se dire analphabète sans que cela soit vraiment le cas et, sous ce prétexte, indiquer son choix en public. Les dominants locaux auraient pu en déduire que ceux parmi leurs dépendants qui préféraient voter en secret leur étaient déjà hostiles. Gladstone maintint donc la nécessité du certificat ${ }^{5}$.

Les Lords ne cédèrent cependant pas et Forster élabora finalement une formule de compromis : l'électeur analphabète devrait faire signer une déclaration certifiant son handicap par un des électeurs de sa circonscription ${ }^{6}$. Simultanément, Gladstone annonça qu'il demanderait une dissolution à la reine si les Lords continuaient de refuser le vote secret. Une partie de la presse conservatrice se montra alors favorable à l'organisation d'élections générales sur cet enjeu. Mais Disraeli et Richmond ne pensaient pas pouvoir en tirer parti et la Chambre des Lords renonça à ses propres amendements le 12 juillet 1872, après trois lectures dans chaque assemblée. La loi finalement adoptée souffrait de nombreuses lacunes mais, en mettant fin au jeu des influences traditionnelles, marquait le déclin irréversible d'un ordre traditionnel dont les Lords étaient les derniers représentants dans le système institutionnel. A la Chambre des Communes, les conservateurs eux-mêmes

1. Ibid., débat du 28 juin 1872 , col. 368 .

2. Ibid., vol. 211, débat du 9 mai 1872, col. 557.

3. Ibid., vol. 212 , débat du 30 mai, col. 847 .

4. Ibid., vol. 211, débat du 13 mai 1872, col. 669-670.

5. Ibid., vol. 212 , débat du 2 juillet 1872 , col. 483 .

6. Ibid., débat du 12 juillet 1872, col. 1045. 
étaient partagés, certains souscrivant au bien-fondé du vote secret $^{1}$. Disraeli, hostile au ballot jusqu'à la fin, plaça d'ailleurs très tôt ces espoirs dans le duc de Richmond et n'intervint que discrètement dans ce débat ultime. Le fait que la Chambre des Lords ait été isolée dans son opposition au ballot illustre bien la dimension sociale de cet enjeu : l'introduction du vote secret vient couronner la montée en puissance des classes moyennes sur la scène publique, comme le souligne O'Leary ${ }^{2}$. Si ces classes moyennes ont surtout dû lutter ici contre l'aristocratie pour obtenir le vote secret, celui-ci aura cependant aussi été perçu, par certains libéraux, comme un instrument dirigé contre les ennemis du futur, les "classes laborieuses" et leurs syndicats.

Cette étude semble suggérer deux séries de conclusions de portée plus générale. Le débat autour du ballot, au cours du XIXe siècle, illustre bien la façon dont les idées circulent, passant d'un camp à l'autre, a fortiori lorsque leurs fondements sont ambigus. L'utilitarisme de Bentham et J. Mill s'est converti au vote secret en faisant violence à l'un de ses principes clés - le principe de publicité - et après avoir élaboré la formule-compromis du vote comme trust. Ces constructions ambivalentes distingueront le radicalisme britannique de la philosophie des Droits de l'Homme et affaiblira la revendication du ballot de deux façons : d'une part, l'utilitarisme fournira des arguments à ses adversaires pour le contrer (le principe de publicité ou l'idée du vote comme trust) ; d'autre part, certains de ces représentants pourront se retourner contre la cause du ballot, comme J. S. Mill, en restant fidèle à certaines prémisses utilitaristes. Le chassé-croisé entre J. S. Mill et Gladstone est d'autant plus intéressant que celui-ci aussi devient partisan du ballot tout en restant fidèle à sa logique en constatant la perte de validité de la conception du vote comme trust. C'est seulement à ce stade que la définition du vote secret comme un droit obtient droit de cité au sommet de l'Etat.

Seconde série de conclusions : les arguments exprimés pour ou contre le vote secret procèdent de motifs à la fois idéologiques et sociaux qu'il est parfois difficile de hiérarchiser. Le cas des utilitaristes est fort instructif à cet égard. La théorie de Bentham tantôt rejetant, tantôt intégrant le vote secret, semble moins sur-déterminée par des intérêts sociaux que par la poursuite d'une obsession de type idéal : le plus grand bonheur du plus grand nombre. A l'inverse, J. Mill revendique explicitement le ballot au nom des ambitions d'une classe moyenne soucieuse de supplanter l'aristocratie qui doit encore une part de son influence à la publicité du vote. Les conservateurs, et au premier rang d'entre eux, une majorité des Lords, combinent aussi des arguments idéologiques (l'attachement soi-disant britannique à un code moral aristocratique valorisant le courage d'une expression publique du vote) et sociaux (la défense d'un système social hiérarchique fondé sur l'inégalité devant la propriété). La déférence constitue ici le point de contact évident entre l'idéologie et l'intérêt social qui s'avèrent fortement imbriqués. Il est souvent difficile, dans les deux cas, de faire la part des choses, de distinguer l'impact de l'intérêt du poids des valeurs à travers lesquelles le monde est vu. A ce titre, le débat sur le ballot illustre sans doute un problème éternel des sciences sociales : l'irréductibilité des valeurs à l'intérêt et vice-versa.

1. O'Leary (C.), The elimination of corrupt practices in British elections 1868-1911, op. cit., p. 85. C'est là le constat de Disraeli lui-même (Ibid., P. 82).

2. Ibid., p. 87. 